Objectives: To investigate factors associated with the development of sepsis in patients with IMIDs using the Taiwanese National Health Insurance Research Database (NHIRD).

Methods: We identified all biologic-naïve patients with RA, AS, PsO, PsA, or CD/ UC from the claim data via the NHIRD who started their first anti-TNF-a agent (etanercept (ETN), adalimumab (ADA) or golimumab (GOL)) between 2003 and 2017 as study subjects. The index date was the first date of anti-TNF-a prescription. Sepsis was defined based on the sepsis-3 definition. We identified sepsis patients using a validated ICD-9-CM coding system proposed by Angus et al, in which a diagnosis of bacterial/fungal infection with one or more acute organ dysfunction is required to define an episode of sepsis. All study subjects were followed up till the date of first hospitalization due to sepsis, 90 days after the last date of anti-TNF-a prescription, withdrawal from NHIRD or death, whichever came first. We used a Cox regression analysis to assess the associations of covariates with the risk of sepsis shown as hazard ratios (HRs) with 95\% confidence interval (Cls). Covariates included anti-TNF-a agent, IMID, age, sex, insured amount, level of urbanization, disease duration, Charlson comorbidity index $(\mathrm{CCl})$, a history of prior hospitalization due to sepsis within 3 months before the index date and medication use within 12 months before the index date and during the follow-up period.

Results: We identified 18105 biologic-naïve patients with IMIDs, including 8123 ETN users, 7623 ADA users and 2359 GOL users. The incidence rates (IRs) of sepsis in patients treated with ETN, ADA and GOL were 1080, 1181, and 617 per $10^{5}$ years respectively. Multivariable regression analyses showed that factors associated with an increased risk of sepsis were use of ADA (ETN as reference: $\mathrm{HR}, 1.21 ; 95 \% \mathrm{Cl}, 1.02-1.42)$, male ( $\mathrm{HR}, 1.24 ; 95 \% \mathrm{Cl}, 1.04-1.48)$, age (HR, 1.06; $95 \% \mathrm{Cl}, 1.05-1.07)$, CD/UC (HR, 2.35; 95\% Cl, 1.57-3.53), CCl (HR, 1.30; 95\% Cl, 1.23-1.38), prior sepsis (HR, 2.42; 95\% Cl, 1.78-3.29), prior use of sulfasalazine (HR, 1.25, 95\% Cl, 1.00-1.55), lower levels of urbanization (level III: HR, 1.37; 95\% $\mathrm{Cl}, 1.06-1.77$; level IV: HR, 1.68, 95\% Cl, 1.35-2.10). Factors associated with a decreased risk of sepsis were use of GOL (ETN as reference: HR, 0.59; 95\% $\mathrm{Cl}, 0.39-0.84)$, use of methotrexate ( $\mathrm{HR}, 0.78 ; 95 \% \mathrm{Cl}, 0.65-1.00)$, and higher insured amount (reference: $\leq 15480$ NTD; 15480-28800 NTD: HR, 0.83; 95\% Cl, $0.68-0.99$; 28800-45800 NTD: HR, 0.58; 95\% Cl, 0.45-0.74; >45800 NTD: HR, $0.33 ; 95 \% \mathrm{Cl}, 0.21-0.54)$

Conclusion: Our study revealed that among biologic-naïve IMID patients initiating anti-TNF-a treatment, use of ADA, age, sex, $\mathrm{CD} / \mathrm{UC}, \mathrm{CCl}$, prior sepsis, prior use of sulfasalazine and lower levels of urbanization were associated with an increased risk of sepsis, while use of GOL, use of methotrexate, and higher insured amount were associated with a decreased risk of sepsis.

Disclosure of Interests: BO-CHUEN HSU: None declared, Hsin-Hua Chen: None declared, Ching-Heng Lin: None declared, Yi-Ming Chen: None declared, KuoLung Lai: None declared, Der-Yuan Chen: None declared, Wen-Nan Huang: None declared, Yi-Hsing Chen Grant/research support from: Taiwan Ministry of Science and Technology, Taiwan Department of Health, Taichung Veterans General Hospital, National Yang-Ming University, GSK, Pfizer, BMS., Consultant of: Pfizer, Novartis, Abbvie, Johnson \& Johnson, BMS, Roche, Lilly, GSK, Astra\& Zeneca, Sanofi, MSD, Guigai, Astellas, Inova Diagnostics, UCB, Agnitio Science Technology, United Biopharma, Thermo Fisher, Gilead., Paid instructor for: Pfizer, Novartis, Johnson \& Johnson, Roche, Lilly, Astra\& Zeneca, Sanofi, Astellas, Agnitio Science Technology, United Biopharma., Speakers bureau: Pfizer, Novartis, Abbvie, Johnson \& Johnson, BMS, Roche, Lilly, GSK, Astra\& Zeneca, Sanofi, MSD, Guigai, Astellas, Inova Diagnostics, UCB, Agnitio Science Technology, United Biopharma, Thermo Fisher, Gilead.

DOI: 10.1136/annrheumdis-2020-eular.1601

\begin{tabular}{|l|l}
\hline OP0070 & THE RISK OF PRECANCEROUS LESIONS OF \\
THE BREAST AND CERVIX UTERI IN PATIENTS \\
WITH AUTOIMMUNE RHEUMATIC DISEASES: A \\
NATIONWIDE POPULATION STUDY IN TAIWAN
\end{tabular}

P. H. Tsai ${ }^{1}$, C. F. Kuo ${ }^{1,2}{ }^{1}{ }^{1}$ Linkou Chang Gung Memorial Hospital, Division of Rheumatology, Allergy and Immunology, Taoyuan City, Taiwan, Republic of China; ${ }^{2}$ University of Nottingham, Division or Rheumatology, Orthopedics and Dermatology, School of Medicine, Nottingham, United Kingdom

Background: Precancerous lesions are pathologically atypical tissues which share part of abnormal features of cancerous tissue under microscopic examination but lack the ability of uninhibited growth and distant metastasis. Some of the measures to screen for precancerous tissues have gained popularity because supporting evidence indicates the beneficial effects on cancer incidence and mortality [1, 2]. However, the roles of these screenings and the risk of precancerous lesions in patients with autoimmune diseases have not been clarified. We aim to exam the risk of precancerous lesions among different autoimmune diseases.

Objectives: To determine whether the Taiwanese autoimmune rheumatic diseases (ARDs) patients have a higher risk of precancerous lesions of the breast and cervix uteri.

Methods: The Taiwan national breast cancer screening program provides biennially mammography for women above 45 years old or with a positive family history of breast cancer above 40 years old. The national cervical cancer screening program provides Pap smear test once every three years for women age above 30 years old. Using the National Health Insurance $(\mathrm{NHI})$ database, we identified a cohort of 6 different groups of ARDs patients in Taiwan between 2004 and 2014 We linked the data from the national screening program of breast and cervical cancer and the $\mathrm{NHI}$ database to estimate the standardized incidence ratios (SIRs) of precancerous lesions (atypical lobular and ductal hyperplasia of the breast, carcinoma in situ of the breast, cervical intraepithelial neoplasia 1 to 3 of cervix uteri and carcinoma in situ of cervix uteri) in patients with ARDs compared with the general population.

Results: From 2004-2014, we identified 64,904 patients with autoimmune dis eases. Table 1 shows the number of patients receiving the screening programs. The standardized incidence ratio (SIR) of precancerous lesion of the breast was elevated in patients with rheumatoid arthritis (SIR, 2.21; $95 \% \mathrm{CI}, 1.21-3.46)$ and Sjögren syndrome (SIR, 5.77; 95\% CI, 2.63-9.85). The incidence of precancerous lesion of cervix uteri was elevated in patients with surveyed ARDs compared with the normal population, particularly for patients with systemic lupus erythematosus (SIR 28.94; 95\% Cl, 24.52-33.70) and inflammatory bowel disease (SIR, 27.50; 95\% CI, 7.40-56.32) (Table 2).

Table 2 Standardized incidence ratio (SIR) of precancerous lesion of the breast and cervix uteri in patients with autoimmune rheumatic diseases

\begin{tabular}{lcc}
\hline & Precancerous lesion of Breast & Precancerous lesion of Cervix \\
\hline & $\operatorname{SIR}(95 \% \mathrm{Cl})$ & $\mathrm{SIR}(95 \% \mathrm{Cl})$ \\
Rheumatoid arthritis & $2.21(1.21-3.46)$ & $5.32(4.25-6.50)$ \\
Systemic lupus erythematosus & $1.34(0.27-2.93)$ & $28.94(24.52-33.70)$ \\
Sjögren syndrome & $5.77(2.63-9.85)$ & $8.32(5.72-11.34)$ \\
Systemic sclerosis & $6.45(0.72-15.54)$ & $7.93(2.90-14.80)$ \\
Vasculitis & 0.00 & $7.68(1.54-16.83)$ \\
Inflammatory bowel diseases & 0.00 & $27.50(7.40-56.32)$
\end{tabular}

Conclusion: Precancerous lesions in the breast and cervix uteri were higher in female patients with ARDs and they should receive stringent screening program. References:

[1] Tabar L, et al. Beyond randomized controlled trials: organized mammographic screening substantially reduces breast carcinoma mortality. Cancer 2001, 91(9):1724-1731.

[2] Peirson L, et al. Screening for cervical cancer: a systematic review and meta-analysis. Syst Rev 2013, 2:35.

Disclosure of Interests: None declared

DOI: 10.1136/annrheumdis-2020-eular.3206

\section{OP0071 1 PREDICTING PERSISTENT HIGH-DOSE OPIOID USE AFTER TOTAL KNEE REPLACEMENT}

C. Gopalakrishnan ${ }^{1}$, J. Franklin ${ }^{1}$, Y. Jin ${ }^{1}$, D. Solomon ${ }^{2}$, J. Katz ${ }^{2,3}$, Y. Lee ${ }^{4}$, P. Franklin ${ }^{5}$, J. Lii ${ }^{1}$, R. J. Desai ${ }^{1}$, S. Kim ${ }^{1} .{ }^{1}$ Brigham and Women's Hospital, Harvard Medical School, Division of Pharmacoepidemiology and Pharmacoeconomics, Boston, United States of America; ${ }^{2}$ Brigham and Women's Hospital, Harvard Medical School, Division of Rheumatology, Immunology and Allergy, Boston, United States of America; ${ }^{3}$ Brigham and Women's Hospital, Harvard Medical School, Department of Orthopedic Surgery, Boston, United States of America; ${ }^{4}$ Northwestern University, Division

Table 1 Breast screen and Pap smear test in patients with autoimmune rheumatic diseases

\begin{tabular}{|c|c|c|c|c|c|c|}
\hline & $\begin{array}{l}\text { Rheumatoid arthritis } \\
(\mathrm{n}=28289)\end{array}$ & $\begin{array}{l}\text { Systemic lupus } \\
\text { erythematosus } \\
\qquad(n=11609)\end{array}$ & $\begin{array}{l}\text { Sjögren } \\
\text { syndrome } \\
(n=17647)\end{array}$ & $\begin{array}{l}\text { Systemic } \\
\text { sclerosis } \\
(n=1489)\end{array}$ & $\begin{array}{l}\text { Vasculitis } \\
(n=3050)\end{array}$ & $\begin{array}{l}\text { Inflammatory bowe } \\
\text { disease }(n=2820)\end{array}$ \\
\hline Female patients & 21199 & 9808 & 15785 & 1043 & 1225 & 1034 \\
\hline Breast screening ( $\mathrm{n}, \%$ ) & $6296(29.70 \%)$ & $1382(14.09 \%)$ & 4737 (30.01\%) & $276(26.46 \%)$ & $67(5.47 \%)$ & $215(20.79 \%)$ \\
\hline Pap smear (n, \%) & $12827(60.51 \%)$ & $6495(66.22 \%)$ & $9961(63.10 \%)$ & $670(64.24 \%)$ & $1114(90.94 \%)$ & $665(64.31 \%)$ \\
\hline
\end{tabular}


of Rheumatology, Chicago, United States of America; ${ }^{5}$ Northwestern University, Department of Medical Social Sciences, Chicago, United States of America

Background: Patients undergoing total knee replacement (TKR) are at increased risk of persistent opioid use and dependence

Objectives: To identify patients with persistent high-dose opioid use after TKR using group-based trajectory models (GBTM) and determine predictors of persistent high-dose opioid users using pre-TKR patient characteristics

Methods: Using US Medicare claims (2010-2014), we identified patients aged $\geq 65$ years who underwent a TKR and had no history of cancer or high-dose opioid use (>25 mean morphine equivalents (MME)/day) in the year prior. All patients were continuously enrolled in Medicare for $\geq 360$ days prior to and $\geq 30$ days after the TKR. To determine opioid filling patterns after the surgery, patients were followed up to 360 days from the day of TKR. We modeled 12 monthly indicators of opioid prescription fills as a continuous (MME/day) variable using a censored normal GBTM and categorized patients into 4 groups. The primary outcome was persistent high-dose opioid use defined as patients in trajectory Group 3 (38.8 MME/day) or Group 4 (22.4 MME/day). We split the data into training (2010-2013 data) and test (2014 data) sets and used logistic regression to predict high-dose opioid use vs low-dose opioid use (Groups 1 and 2) as a binary outcome utilizing pre-TKR patient characteristics as candidate predictors using the least absolute shrinkage and selection operator (LASSO) regression for variable selection. A reduced model with only 10 pre-specified variables readily available for clinical use was also considered

Results: The final study cohort included 142,089 patients. The GBTM identified 4 distinct trajectories (Group 1- Short-term, low-dose, Group 2- long-term, low-dose, Group 3- medium-term, high-dose, Group 4-long-term, high-dose) of opioid use in the year after TKR (Figure). Using logistic regression and LASSO, we predicted the probability of persistent high-dose opioid use $(\mathrm{N}=17,171)$ (vs. low-dose opioid use) in the training set $(\mathrm{N}=101,810)$ for an $A \cup C=0.80$. The AUC in the test set $(\mathrm{N}=40,279)$ predicting high opioid use $(\mathrm{N}=5,893)$ was 0.77 . The final model selected 33 variables and identified baseline history of opioid use as the strongest positive predictor of high-dose persistent opioid use. The reduced model with only ten predictors also performed equally well (AUC=0.77) (Table).

Conclusion: In this cohort of older patients with no history of cancer or highdose opioid use at baseline, $16.2 \%$ became high dose (28.1 MME/day) opioid users during the year after TKR. Our prediction model with 10 readily available clinical factors may help identify patients at high risk of future adverse outcomes from persistent opioid use and dependence after TKR

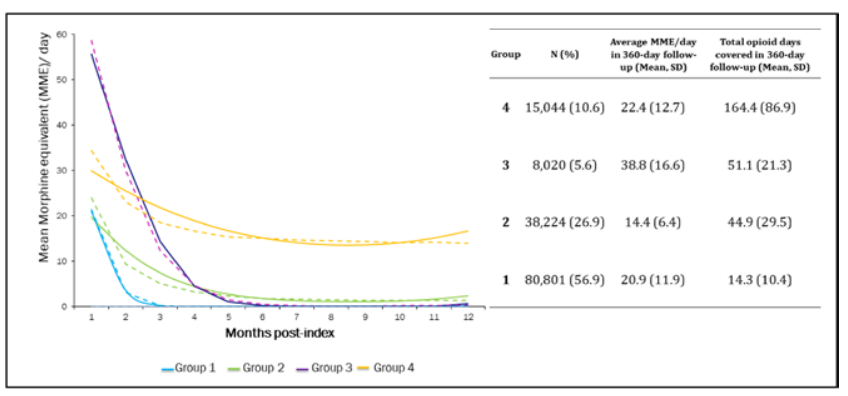

Figure. Trajectories of opioid use patterns after TKR

Table. Predictors of persistent high-dose opioid use in the reduced model

\begin{tabular}{lcc}
\hline Variable & $\begin{array}{c}\text { Multivariable Odds Ratio }(95 \% \mathrm{Cl}) \\
\text { Predicting High dose vs. } \\
\text { Low dose opioid use }\end{array}$ & P-value \\
\hline Age (in years) & $0.94(0.93-0.94)$ & $<0.001$ \\
Females (Ref=Males) & $0.99(0.93-1.06)$ & 0.78 \\
White race (Ref=Other) & $1.25(1.04-1.50)$ & 0.02 \\
Baseline opioid use (MME/day) & $1.22(1.22-1.23)$ & $<0.001$ \\
Substance use (Yes/No) & $1.10(1.02-1.20)$ & 0.02 \\
Benzodiazepine use (Yes/No) & $1.22(1.12-1.32)$ & $<0.001$ \\
Anxiolytic use (Yes/No) & $1.30(1.19-1.43)$ & 0.001 \\
Anticonvulsant use (Yes/No) & $0.94(0.87-1.03)$ & 0.19 \\
Antidepressant use (Yes/No) & $1.03(0.96-1.11)$ & 0.04 \\
NSAID use (Yes/No) & $1.07(1.00-1.14)$ & \\
\hline
\end{tabular}

Disclosure of Interests: Chandrasekar Gopalakrishnan: None declared, Jessica Franklin: None declared, Yinzhu Jin: None declared, Daniel Solomon Grant/ research support from: Funding from Abbvie and Amgen unrelated to this work, Jeffrey Katz Grant/research support from: Dr Katz reported receiving grants from Samumed and Flexion Therapeutics outside the submitted work., Yvonne Lee
Shareholder of: Cigna-Express Scripts, Grant/research support from: Pfizer, Consultant of: Highland Instruments, Inc., Patricia Franklin: None declared, Joyce Lii: None declared, Rishi J Desai Grant/research support from: Dr. Desai reported receiving grants from Bayer, Novartis, and Vertex Pharmaceuticals outside the submitted work., Seoyoung Kim Grant/research support from: Seoyoung $\mathrm{C}$ Kim has received research grants from AbbVie, Roche, Bristol-Myers Squibb and Pfizer.

DOI: 10.1136/annrheumdis-2020-eular.4017

\section{OP0072 \\ IDENTIFYING POLYMYALGIA RHEUMATICA RELAPSE AND ITS ASSOCIATIONS IN A RETROSPECTIVE COHORT}

T. Bolhuis ${ }^{1}$, D. Marsman ${ }^{1}$, N. Den Broeder ${ }^{1}$, F. Van den Hoogen ${ }^{1,2}$, A. Den Broeder ${ }^{1,2}$, A. Van der Maas'. ' 'Sint Maartenskliniek, Rheumatology, Nijmegen, Netherlands; ${ }^{2}$ Radboudumc, Rheumatology, Nijmegen, Netherlands

Background: Polymyalgia rheumatica (PMR) relapse occurs frequently when tapering glucocorticoids $(\mathrm{GC})^{1}$. Some risk factors for varying definitions of relapse have been identified, although with conflicting results ${ }^{2}$. Identification of relapse and its associations can help identify patients in need of tighter follow up or additional medication.

Objectives: To identify PMR GC-taper related relapse (proportion 1 and 2 years after starting treatment and per year of treatment) and candidate predictors, for a future prediction model.

Methods: In a retrospective cohort of new PMR patients, visiting our hospital from April 2008 - January 2018, all visits $>30$ days after starting GC treatment and with $>2.5 \mathrm{mg}$ oral prednisolone were used to identify substantial relapses. Relapse was defined in two ways: rheumatologist judgement (RJ) and treatment intensivation ( $\mathrm{TI})$. Agreement between $\mathrm{RJ}$ and $\mathrm{TI}$ at visits was assessed. TI relapse was used going forward for treatment based prediction. The proportion of relapsers after 1 and 2 years (cumulative incidence) and the amount of relapses per year of treatment (incidence rate (IR)), were assessed. Unadjusted associations with candidate predictors, present when starting GC treatment, were assessed using logistic and Poisson regression respectively.

Results: Data from 417 patients was used (figure 1). Relapse occurred at 405 and 325 (of 2455) visits based on RJ and TI respectively. TI relapse (cumulative incidence) after 1 and 2 years was $134(32 \%)$ and 184 (44\%) and IR was 0.35 per patient year. Unadjusted significant associations for the cumulative incidence were CRP and ESR at baseline, and symptom duration before treatment (table 1), but only CRP and ESR were significantly associated with yearly IR.

Table 1: Associations between predictors and relapse after 1 and 2 years of treatment and the amount of relapses per year of treatment.

\begin{tabular}{|c|c|c|c|c|c|c|c|c|c|}
\hline \multirow{3}{*}{$\begin{array}{l}\text { Predictors } \\
\text { Age - years }\end{array}$} & \multicolumn{3}{|c|}{ Within first year } & \multicolumn{3}{|c|}{ Within first 2 years } & \multicolumn{3}{|c|}{ Incidence rate } \\
\hline & \multirow{2}{*}{$\begin{array}{l}\text { OR } \\
1.00\end{array}$} & \multicolumn{2}{|c|}{$\mathrm{Cl}$} & \multirow{2}{*}{$\begin{array}{l}\text { OR } \\
1.00\end{array}$} & \multicolumn{2}{|c|}{$\mathrm{Cl}$} & \multirow{2}{*}{$\begin{array}{l}\text { IRR } \\
1.00\end{array}$} & \multicolumn{2}{|c|}{$\mathrm{Cl}$} \\
\hline & & 0.98 & 1.03 & & 0.98 & 1.02 & & 0.99 & 1.01 \\
\hline Sex - ref. Male & 1.44 & 0.95 & 2.20 & 1.38 & 0.94 & 2.05 & 1.38 & 0.79 & 1.27 \\
\hline \multicolumn{10}{|l|}{ Medical history - ref. No } \\
\hline - inflammatory & 1.18 & 0.75 & 1.86 & 1.00 & 0.65 & 1.53 & 1.00 & 0.95 & 1.60 \\
\hline - malignancy & 0.69 & 0.36 & 1.35 & 0.96 & 0.53 & 1.73 & 0.96 & 0.64 & 1.35 \\
\hline - cardiovascular & 1.68 & 0.07 & 2.95 & 1.74 & 1.00 & 3.03 & 1.74 & 0.89 & 1.66 \\
\hline \multicolumn{10}{|l|}{ Smoking - ref. no, n=336 } \\
\hline Stopped & 0.85 & 0.49 & 1.46 & 1.16 & 0.70 & 1.92 & 1.16 & 0.96 & 1.74 \\
\hline Yes & 0.81 & 0.412 & 1.57 & 0.98 & 0.53 & 1.82 & 0.98 & 0.67 & 1.40 \\
\hline $\begin{array}{l}\text { Symptom duration before } \\
\text { baseline }- \text { per week, } n=411\end{array}$ & 0.98 & 0.96 & 0.998 & 0.98 & 0.964 & 0.997 & 0.98 & 0.98 & 1.00 \\
\hline $\begin{array}{l}\text { Clinical disease severity - } \\
\text { score } 0-8\end{array}$ & 0.93 & 0.82 & 1.07 & 0.93 & 0.82 & 1.05 & 0.93 & 0.87 & 1.02 \\
\hline $\begin{array}{l}\text { (Suspected) presence of } \\
\text { peripheral arthritis - ref. No }\end{array}$ & 0.73 & 0.40 & 1.33 & 0.67 & 0.38 & 1.16 & 0.67 & 0.55 & 1.16 \\
\hline $\begin{array}{l}\text { Presence of systemic } \\
\text { symptoms - ref. No }\end{array}$ & 0.78 & 0.51 & 1.19 & 0.84 & 0.57 & 1.25 & 0.84 & 0.79 & 1.28 \\
\hline CRP - per $\mathrm{mg} / \mathrm{L}, \mathrm{n}=363$ & 1.01 & 1.00 & 1.01 & 1.01 & 1.00 & 1.01 & 1.01 & 1.00 & 1.01 \\
\hline ESR - per mm/h, $n=396$ & 1.02 & 1.01 & 1.03 & 1.02 & 1.01 & 1.03 & 1.02 & 1.00 & 1.01 \\
\hline $\mathrm{Hb}-$ per mmol/L, $\mathrm{n}=316$ & 1.03 & 0.75 & 1.39 & 0.99 & 0.74 & 1.32 & 0.99 & 0.86 & 1.25 \\
\hline APR - ref. normal, $n=376$ & 0.99 & 0.41 & 2.40 & 0.79 & 0.34 & 1.83 & 0.79 & 0.63 & 1.68 \\
\hline
\end{tabular}

Ref., reference category; $\mathrm{Hb}$, haemoglobin; $\mathrm{APR}$, acute phase reactants; $\mathrm{Cl}, 95 \%$ confidence interval

Conclusion: PMR relapse while tapering GC occurs frequently, and some - although weak - associations were found. Longer symptom duration before treatment decreased chance of relapse, but did not increase the amount of relapses per year of treatment, potentially indicating a more self-limiting disease course. A uniform definition of relapse and identifying further predictors for a potential prediction model is needed to focus GC sparing agents for patients. 